\title{
On the performance of Bluetooth and IEEE 802.15.4 radios in a body area network
}

\author{
Rahul C. Shah \\ Intel Corporation \\ rahul.c.shah@intel.com
}

\author{
Lama Nachman \\ Intel Corporation \\ lama.nachman@intel.com
}

\author{
Chieh-yih Wan \\ Intel Corporation \\ chieh-yih.wan@intel.com
}

\begin{abstract}
The last few years have seen the emergence of many applications such as wellness, chronic disease management and assisted living that require pervasive sensing of people and the environment. Many of these applications require on-body sensing of various parameters including heart-rate, caloric burn, activity, temperature, etc. Low power wireless technologies are a key enabler for these applications, as it allows distributed sensing and aggregation without the cost of wiring the individual. Bluetooth is a well established low power wireless technology and has the advantage of being integrated into many handheld devices today whereas IEEE 802.15.4 has gained momentum in wireless sensor networks over the last few years due to its low power and cost. The performance of these radios in the context of WSN applications has been explored and published in numerous papers. However there hasn't been a lot of work exploring the effect of the human body on the performance of these radios. We have designed and conducted experiments on multiple people to measure the effect of the human body on the performance of Bluetooth and IEEE 802.15.4. We have explored different activities (sitting, standing and walking) as well as many sensor locations (ear, chest, waist, knee and ankle). Finally we explored the co-existence of both of these radios. In this paper, we present the results of these experiments and provide a detailed analysis of the suitability of these radios for body area networks.
\end{abstract}

\section{Keywords}

Body area networks, sensor networks, performance evaluation.

\section{INTRODUCTION}

The last few years have seen the emergence of many applications such as wellness, chronic disease management and assisted living that require pervasive sensing of people and the environment. Many of these applications require on-body sensing of various parameters including heart-rate, caloric burn, activity, temperature, etc. Wireless technologies are a key enabler for these applications, as it allows distributed sensing and aggregation without the cost of wiring the individual. There are many wireless technologies available today, that cover a wide range of capabilities and cost. At the higher end of the spectrum, IEEE

Permission to make digital or hard copies of all or part of this work for personal or classroom use is granted without fee provided that copies are not made or distributed for profit or commercial advantage and that copies bear this notice and the full citation on the first page. To copy otherwise, to republish, to post on servers or to redistribute to lists, requires prior specific permission and/or a fee.

BodyNets 2008, March 13-15 Tempe, Arizona, USA

Copyright (C) 2008 ICST 978-963-9799-17-2

DOI 10.4108/ICST.BODYNETS2008.2972
802.11 provides data rates in the tens of megabits per second at power consumption rates of hundreds of $\mathrm{mW}$. At the lower end of the spectrum, IEEE 802.15.4 has gained a lot of momentum over the last few years in the wireless sensor networks space due to its low power $(\sim 50 \mathrm{~mW}$ range $)$ and acceptable data rates $(256 \mathrm{kbps}$ max). Bluetooth is somewhere in the middle with a maximum data rate of $768 \mathrm{kbps}$ and power consumption in the $90 \mathrm{~mW}$ range, however, it has the advantage of much wider adoption and integration into most hand-held devices today.

Typically, most applications in the health domain require continuous sensing of the individual while at the same time having stringent constraints on the battery size of the sensing nodes to enable the wearability of these devices. In addition, many of these sensors have low data rate requirements, with ECG being one of the most demanding ( $4 \mathrm{kbps}$ per lead). As a result, we focus on IEEE 802.15.4 and Bluetooth in this paper as they provide a more suitable tradeoff between data rates and power consumption than higher power radios like IEEE 802.11. There is a large body of work analyzing the performance of IEEE 802.15.4, and to a lesser extent Bluetooth, in the context of wireless sensor networks. However, the effect of the human body and mobility on these radios has not been studied as extensively, and to our knowledge, there is no work comparing Bluetooth and IEEE 802.15.4 in these environments.

On-body sensing architectures are usually comprised of multiple tiers. At the first tier, small low power sensing devices are attached to different locations on the body including chest (e.g. ECG), ankle or knee (e.g. accelerometer), arm (e.g. temp, heat), ear $(\mathrm{SpO} 2)$, etc. These nodes typically form a star network with an on-body aggregator device (tier 2) where data can be aggregated, processed and relayed to backend servers (tier 3 ). The human body poses multiple challenges to the electromagnetic propagation of these radios due to the higher attenuation of the human tissue and the rapidly changing environment resulting from mobility and different body postures. To evaluate the effect of these factors and the suitability of these radios for on-body propagation, we conducted multiple experiments and measured data throughput and packet error rates in these different scenarios. First, we explored different locations on the body using the expected sensor locations including waist, ankle, ear, knee and chest. We assumed the on-body aggregator to be located on the waist (typical scenario). We compared these results to off-body configurations to isolate the on-body effect. We also varied the activity of the person between standing, sitting and walking to estimate the effect of mobility and posture. We conducted the above experiments for IEEE 802.15.4 and Bluetooth and compared the resilience of both of these radios to these factors. Finally, we explored the effect of co-existence of these radios.

*Other names and brands may be claimed as the property of others. 
The rest of the paper is organized as follows. Section 2 compares and summarizes related work. Section 3 describes the experiment methodology and explains the selection of the evaluation metrics. Section 4 presents the experimental results for both IEEE 802.15.4 and Bluetooth. Section 5 provides an in depth analysis of the experimental results. Finally Section 6 concludes the paper and describes future work.

\section{RELATED WORK}

There has been a considerable amount of work done in investigating the RF performance and propagation models at 2.4 GHz. Chui [1] proposed a discrete event simulation technique to model the performance of a Bluetooth piconet under variable loading and bit error rate (BER) conditions due to physical layer impairments such as human body obstruction, multipath fading, etc. Roelens, etc. [2], developed a propagation model for RF communication $5-40 \mathrm{~cm}$ above human tissue. The model was applied to muscle and brain simulating media and validated by simulations and measurements. The energy absorption mechanism by biological bodies was studied in [3] via numerical simulations. In [5], the authors proposed a propagation loss model for communication within the human body and verified the model for the frequency range from $900 \mathrm{MHz}$ to $3 \mathrm{GHz}$ using 3D EM simulation software. Johansson [4] studied the radiation patterns and their dependencies on body size and posture through simulation.

All aforementioned studies approximate the human body through simulations or analytical modeling and only evaluate the impact on wireless communications at the physical layer in some generic portion of the RF spectrum. While these studies are invaluable in understanding the fundamental behavior of EM radiation around/in human body, they do not offer enough engineering insights for system designers to build prototype systems utilizing commercially available wireless solutions for medical applications. It is our intention to fill this gap through this paper.

Among similar efforts, there are a number of groups looking at the performance and coexistence issue between various radio standards sharing the ISM band. In [6], the authors examined the coexistence between IEEE 802.15.4 devices in peer-to-peer (unslotted) mode through simulations on OPNET. Werb et. al. [7] studied the performance of IEEE 802.15.4 in industrial facilities such as a machine room or compressor house. Their results showed that the link quality in those environments could be substantially improved with a protocol that utilized multiple frequencies to combat the frequency selective fading effect. They also observed that IEEE 802.15.4 radio at very low power will suffer from serious interference from WiFi and Bluetooth. In [8] the authors examined the high level coexistence performance between IEEE 802.15.4, WiFi, Bluetooth and microwave ovens using off-the-shelf available equipment and software without any adaptation. Their result showed that IEEE 802.15.4 radio is generally robust against interference of other $2.4 \mathrm{GHz}$ systems mentioned above. Note, however, that none of these works look at the fading effect of the human body on these radios.

A number of research projects [9] [10] [11] [12] are exploring medical sensor networks. CodeBlue [12] is one of the first integrated solutions that combine hardware and software platform for medical sensor networks application. The authors evaluated the system through an indoor testbed of 30 fixed nodes (with
IEEE 802.15.4 radio) and presented results on its scalability, fairness, data delivery latency and mobility effect. They identified two major communication problems, i.e., 1) the lack of reliable communication and 2) the impact of bandwidth limitation on IEEE 802.15.4. While these observations are consistent with our results, they did not evaluate Bluetooth, a radio technology we found superior in addressing the two communication problems identified in their work. Furthermore, even though the authors presented results on the mobility effect, their wireless nodes were mounted on laptops not on humans.

More closely related to our effort is the work described in [13]. The authors examined the performance of IEEE 802.15.4 through and around the human body using network layer metrics such as packet delivery ratio and latency. They observed that the human body is similar to aluminum in that it is a very good RF shield such that that no packet can get through without utilizing multipath. The authors also developed a useful visualization technique to discern communication patterns in large data sets visually. Note that we have borrowed the same visualization technique in presenting most of our data sets in this paper. The main focus of the above paper, however, was on evaluating the network topology (star versus multi-hop) against design goals such as power and delay efficiency.

\section{EXPERIMENT METHODOLOGY}

As mentioned previously, we tested the performance of IEEE 802.15.4 (802.15.4) and Bluetooth (BT) for nodes placed at five positions on the body - right ear, chest (above the heart), left side of the waist, right knee and right ankle. The aggregator node was placed on the right side of the waist to mimic a typical usage scenario. We measured the performance for data sent in both directions - to and from the aggregator node. However, due to lack of space, we mostly present the performance data for the direction towards the aggregator as we envision this to be the prevalent traffic pattern for most medical use cases. The one exception is the case where we look at some specific differences in performance between the two directions.

The experiments were typically carried out while our volunteers (test subjects who carry the body area network on their body) were sitting, standing and walking to identify the effect of mobility and body position on the performance. However, some experiments were only carried out while in the sitting position (considered as the default position), which will be noted as appropriate. Furthermore, most of the experiments were carried out on 3 different people for 802.15.4 and BT (a total of 5 people were used, with one common person for both radios), with the exceptions noted in the results section when the experiment was only done on a single person. The experiments were carried out indoors in typical office and home environments.

Most health applications require the sensing of either scalar data such as blood oxygen saturation or streaming data such as ECG; the former typically results in small packets while the latter results in large packets (usually dictated by the maximum packet size allowed by the radio). Since the maximum packet size for 802.15 .4 is 127 bytes, we chose 100 bytes as the larger packet size and 10 bytes as the small packet size and used those to compare both the radios. We also ran one experiment for Bluetooth with 300 byte packets (maximum packet size is 339 bytes) to evaluate 


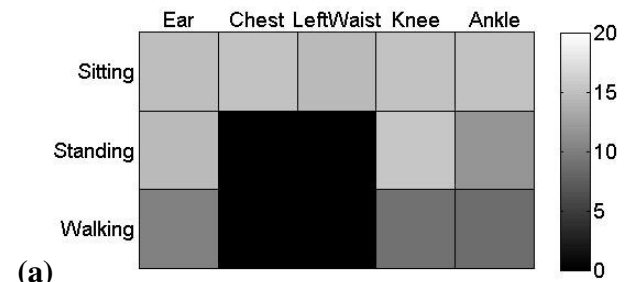

(a)

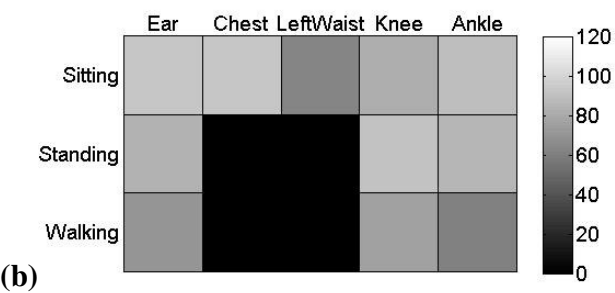

Figure 1 Goodput (in kbps) for 802.15.4 using (a) 10 byte packets and (b) 100 byte packets. Black squares indicate no data is available for that case.

its performance for larger packets. Obviously we cannot directly compare this packet size with 802.15.4.

\subsection{Hardware and software used}

Intel Motes [14] were used as the hardware platform to evaluate the performance of Bluetooth. They consist of an off-the-shelf Zeevo module that integrates an ARM processor and a Bluetooth chipset. The device runs TinyOS [16], an event-driven operating system popular in the sensor networking community. To test the 802.15.4 radio, Intel Mote 2's [15] were used. These consist of an XScale PXA271 processor and a CC2420 [17] IEEE 802.15.4 radio. These motes also run TinyOS. It is important to note that the Bluetooth protocol we used was faithful to the standard (BT 1.1), but the 802.15.4 protocol we used was B-MAC [18] which is a MAC included with TinyOS and is similar but not identical to the actual 802.15.4 standard. Note that many of the Bluetooth sensing applications leverage the serial port profile (SPP), however, we decided to stay at the MAC layer to avoid introducing more uncertainty due to the network and transport layers. Hence, we implemented our application directly over HCI in the case of Bluetooth and BMAC in the case of 802.15.4. Both Bluetooth and IEEE 802.15.4 radios were set to transmit at 0 $\mathrm{dBm}$. The antennas used were $2.4 \mathrm{GHz}$ surface mount antennas.

The software to test the radio performance was mostly identical on both platforms (the only difference was in the radio specific calls). A typical experiment consisted of one node attached to a computer via USB as the base station that would receive commands from the computer and send them via the radio. These commands would be received by the sender and receiver nodes on the body which would then start the required experiment. Most experiment runs lasted for 5 minutes. During the experiment, packets were sent back-to-back and the receiver node would keep track of all the packets it received or missed (using sequence numbers) and write all this data to the internal flash. This data could then be downloaded at a later time for analysis. Note that once the sending and receiving nodes were commanded to start an experiment, the on-body devices were completely autonomous and there was no need to be near a computer, enabling testing for walking scenarios. Furthermore, in the case of the Bluetooth experiments, the commanding node was switched off once the (a)

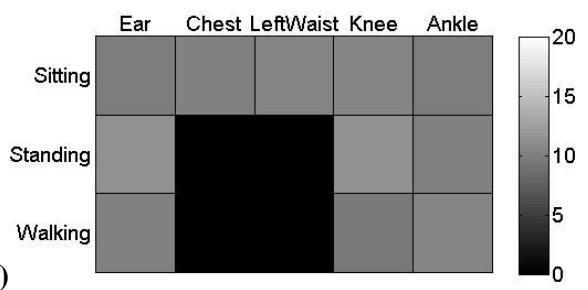

(b)

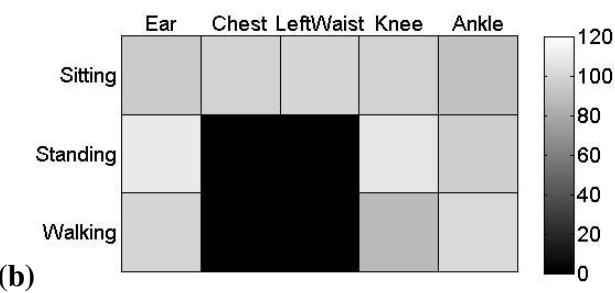

Figure 2 Goodput (in kbps) for BT using (a) 10 byte packets and (b) 100 byte packets. Black squares indicate no data is available for that case

experiment was set up and didn't participate in the piconet during the execution of the experiment. This was done to ensure that the commanding node did not affect the bandwidth by consuming any of the slots.

\subsection{Performance metrics}

The data logged during an experiment was used to calculate three performance metrics which we present in this paper:

- Packet success rate: This is the fraction of packets sent that were successfully received at the destination.

- Goodput: This is the number of bits per second correctly received at the receiver.

- Latency: This is the time since a packet is ready for transmission at the sender until the time it is successfully received at the destination.

Note that the IEEE 802.15.4 protocol does not mandate automatic retransmission, whereas the $\mathrm{BT}$ radio has a reliable link layer that automates acknowledgments and retransmission. This resulted in BT having $\sim 100 \%$ success rate in our tests. Moreover, the latency we measured in the case of BT would include the retransmission time (if necessary) till the packet was successfully received. On the other hand, since most of the 802.15.4 tests did not include acknowledgments and retransmissions, the measured latency would be on the smaller side due to less overhead during the experiments.

\section{EXPERIMENTAL RESULTS}

This section presents the results obtained from the experiments mentioned in the previous section. Each of the subsections below will compare 802.15.4 and Bluetooth radios for specific scenarios. All 802.15.4 experiments were carried out at channel 11.

\subsection{Baseline (off-body) results}

The first set of experiments was conducted between two nodes placed $1 \mathrm{~m}$ apart on a table to establish the baseline performance. Comparing these results with the subsequent on-body results provides a better idea of the effect of the human body compared to other external environmental factors. The choice of $1 \mathrm{~m}$ as the 


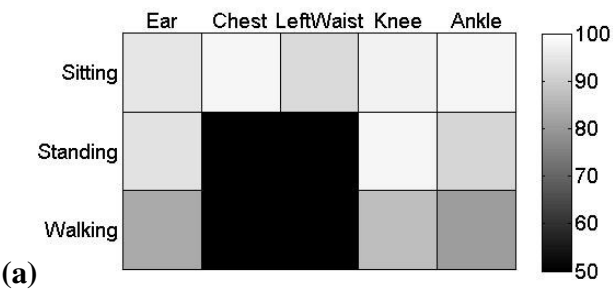

(a)

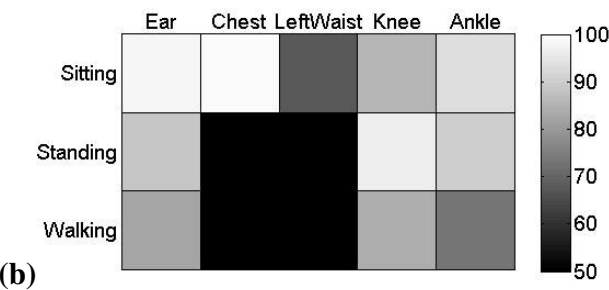

Figure 3 Packet success rate (in \%) for 802.15.4 using (a) 10 bytes and (b) 100 bytes packets. Black squares indicate no data is available for that case.

distance between the two nodes was dictated by the rough distance between nodes placed on an extremity and the waist of an average person's body. The results for the baseline case for 802.15.4 radios are presented in Table 1 .

Table 1 Baseline (off-body) results for different packet sizes for 802.15.4 radios when sender and receiver are $1 \mathrm{~m}$ apart

\begin{tabular}{l|lll}
\hline $\begin{array}{l}\text { Packet } \\
\text { size } \\
\text { (bytes) }\end{array}$ & $\begin{array}{l}\text { Goodput } \\
(\text { kbps })\end{array}$ & $\begin{array}{l}\text { Success } \\
\text { rate }(\%)\end{array}$ & $\begin{array}{l}\text { Mean } \\
\text { latency } \\
(\mathrm{ms})\end{array}$ \\
\hline 10 & 16.43 & 98.94 & 4.82 \\
100 & 94.63 & 99.78 & 8.44 \\
\hline
\end{tabular}

(a)
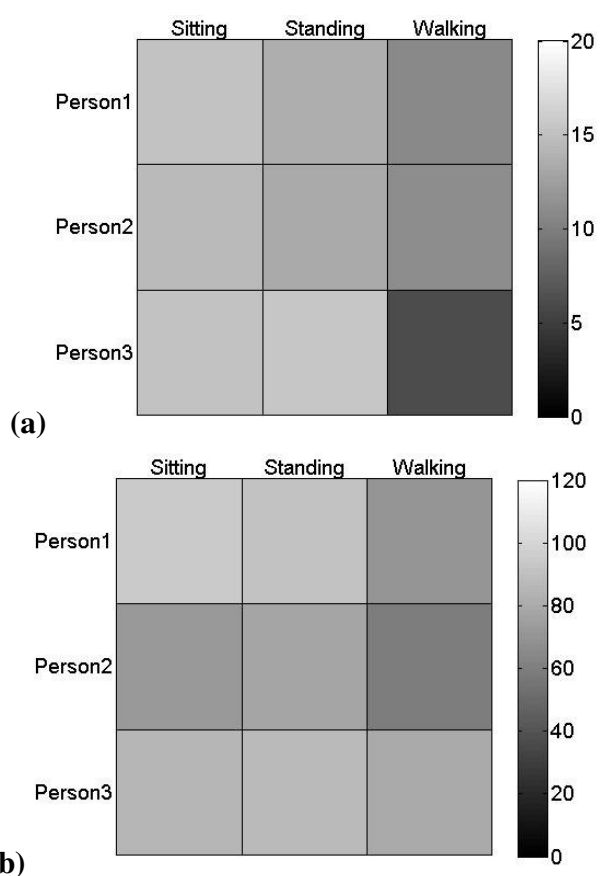

(b)

Figure 4 Goodput (in kbps) for 802.15.4 using (a) 10 byte packets and (b) 100 byte packets.
For the Bluetooth case, the baseline (off-body) results are presented in Table 2. As can be seen, IEEE 802.15.4 has higher goodput for smaller packet sizes, whereas Bluetooth has better goodput for larger packets. The much lower goodput for 10 byte packets in Bluetooth is caused by the fact that this payload would not fit in a single slot packet due to the extra headers we are currently using, hence at best it will use a 3-slot packet, resulting in wasted bandwidth. Note the 3-slot packet does accommodate the 100 byte payload.

Table 2 Baseline (off-body) results for different packet sizes for BT radios when sender and receiver are $1 \mathrm{~m}$ apart

\begin{tabular}{l|lll}
\hline $\begin{array}{l}\text { Packet } \\
\text { size } \\
\text { (bytes) }\end{array}$ & $\begin{array}{l}\text { Goodput } \\
(\mathrm{kbps})\end{array}$ & $\begin{array}{l}\text { Success } \\
\text { rate }(\%)\end{array}$ & $\begin{array}{l}\text { Mean } \\
\text { latency } \\
(\mathrm{ms})\end{array}$ \\
\hline 10 & 12.36 & 100 & 6.47 \\
100 & 115.03 & 100 & 6.95 \\
300 & 232.2 & 100 & 10.34 \\
\hline
\end{tabular}

\subsection{On-body results (single sender)}

Figure 1 shows the results for the goodput of IEEE 802.15.4 when 10 byte or 100 byte packets are sent back-to-back. The figure shows the goodput for a combination of different activities and node positions averaged over 3 people. Corresponding results for Bluetooth are shown in Figure 2. The first thing that can be noted for Bluetooth is that the goodput is much more consistent across different node positions and activities. The second thing is that the goodput for Bluetooth is lower than 802.15 .4 for the 10 byte packets, whereas it is higher for the 100 byte packets. This result highlights the overhead penalty in Bluetooth for smaller packets and is similar to the baseline case.

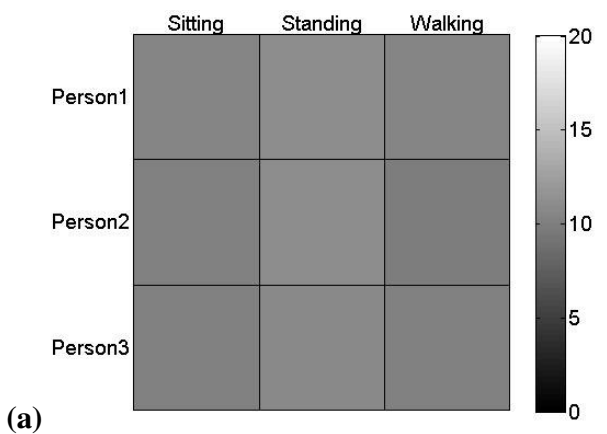

(a)

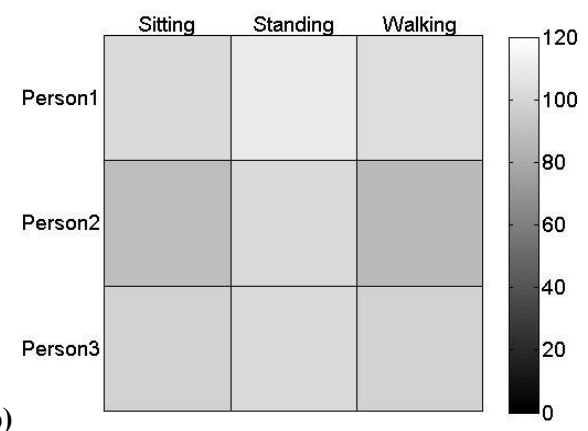

Figure 5 Goodput (in kbps) for BT using (a) 10 byte packets and (b) 100 byte packets. 


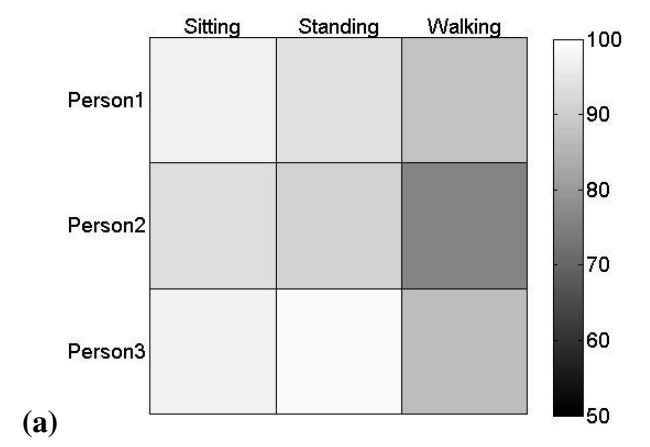

(a)

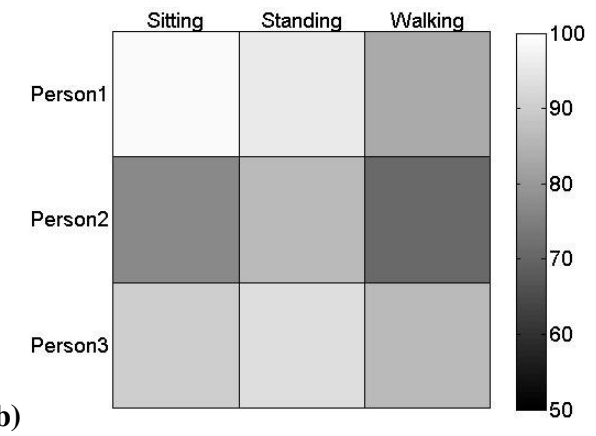

Figure 6 Packet success rate (in \%) for 802.15.4 using (a) 10 bytes and (b) 100 bytes packets.

The packet success rates for the same experiment runs for 802.15.4 are shown in Figure 3. This clearly shows the worse performance as the person is walking vs. sitting or standing. Additionally, there are certain cases when the performance is worse, for e.g. from the left waist to the waist for 100 byte packets, for which we do not know a reason. Correspondingly for Bluetooth, the packet success rate is $100 \%$ due to automatic retransmissions, hence those results are not shown here. Note that the black squares in Figures 1-3 indicate that no data was available for those cases.

Figure 4 compares the goodput for 802.15.4 for different people

(a)

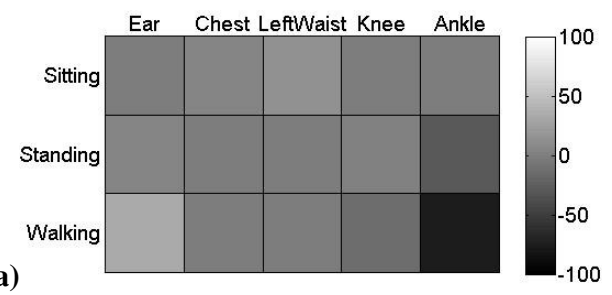

(b)

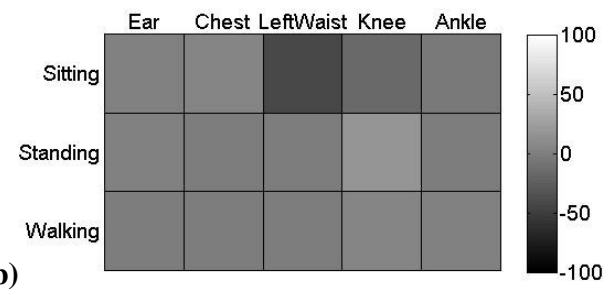

Figure 7 Difference in the goodput for 802.15.4 to and from the waist as a percentage of the goodput to the waist for packet sizes of (a) 10 bytes and (b) 100 bytes. Note that the colors closer to the value zero indicate better symmetric links while lighter or darker colors indicate more asymmetric links. and different activities averaged over all node positions. The plot of the corresponding packet success rates are shown in Figure 6. It can be clearly seen that walking has somewhat worse performance than sitting or standing. The performance also varies quite a bit across different people. Similarly, Figure 5 shows the goodput results for different people and activities for Bluetooth. Again, the performance is fairly consistent across different activities and people. Since the packet success rates were $100 \%$ in all cases for Bluetooth, those results are not shown.

Another point to be compared is the difference in goodput from or to a node on the waist. This would indicate if there is an asymmetric channel. This is presented in Figure 7 for packet sizes of 10 and 100 bytes for 802.15.4. The results are for different activities and node positions averaged over 3 different people. This shows that the channels are mostly symmetric and there is no definite pattern of asymmetry, though some node position activity combinations exhibited asymmetry (for e.g. the case of ankle-walking and ear-walking for 10 byte packets and left waistsitting for 100 byte packets showed up as an asymmetric channel due to some particularly bad experiment runs). Similar to 802.15.4, the results for Bluetooth are shown in Figure 8. In general, the channel seems to be fairly symmetric, with a couple of experiment runs causing differences in performance that show up as an asymmetric channel.

Table 3 Average latency (ms) for various node positions for 802.15.4

\begin{tabular}{l|ll}
\hline & $\begin{array}{c}\text { 10 byte } \\
\text { packets }\end{array}$ & $\begin{array}{c}\text { 100 byte } \\
\text { packets }\end{array}$ \\
Ear & 5.45 & 8.48 \\
Knee & 7.08 & 8.47 \\
Ankle & 7.17 & 8.47 \\
\hline
\end{tabular}

Table 3 shows the results for the mean latencies (in ms) for 10

(a)

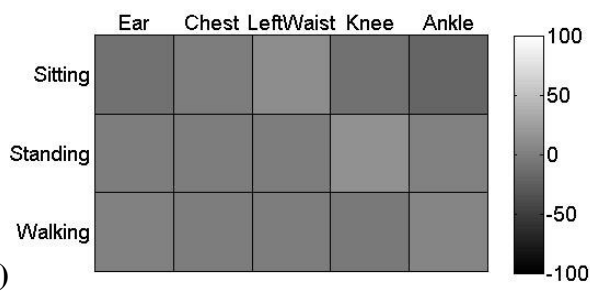

(b)

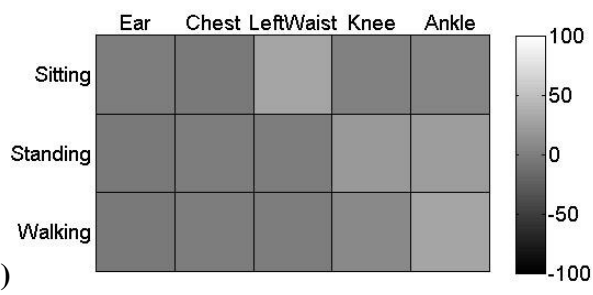

Figure 8 Difference in the goodput for Bluetooth to and from the waist as a percentage of the goodput to the waist for packet sizes of (a) 10 bytes and (b) 100 bytes. Note that the colors closer to the value zero indicate better symmetric links while lighter or darker colors indicate more asymmetric links. 
byte and 100 byte packets for different node positions for 802.15.4. The result for each node position is averaged over all people and activities. It is interesting to note that the latency was constant for all node positions for the 100 byte packets, but was much smaller for the ear compared to the knee and ankle for the 10 byte packets. The latency results for Bluetooth are shown in Table 4. As can be seen, the mean latencies are fairly consistent across different node positions and packet sizes.

Table 4 Avg. latency (ms) for different node positions for BT

\begin{tabular}{l|ll}
\hline & $\begin{array}{c}\text { 10 byte } \\
\text { packets }\end{array}$ & $\begin{array}{c}\text { 100 byte } \\
\text { packets }\end{array}$ \\
\hline Ear & 7.69 & 7.97 \\
Knee & 7.68 & 8.39 \\
Ankle & 7.92 & 8.56 \\
\hline
\end{tabular}

We can also look at the sequences of missed packets from the experiment runs to identify the average and worst case channel fade durations. This is presented in Table 5 for 802.15.4. While the mean fade duration is $<20 \mathrm{~ms}$, the worst case can be as long as 10 seconds. This clearly calls for substantial buffering requirements at the nodes if reliability of the data is important. Similar data is not available for Bluetooth since missed packet information is not available at the application layer due to automatic retransmission.

Table 5 Average and maximum duration of channel fade (missed packets) for 802.15.4 for different node positions

\begin{tabular}{l|ll}
\hline & $\begin{array}{c}\text { Avg. fade duration } \\
\text { (in ms) }\end{array}$ & $\begin{array}{c}\text { Max. fade duration } \\
\text { (in ms) }\end{array}$ \\
\hline Ear & 15.48 & 10640 \\
Knee & 15.32 & 7377 \\
Ankle & 17.08 & 6758 \\
\hline
\end{tabular}

\subsection{On-body results (multiple senders)}

All the results in the previous section were for a single node sending data to another node on the body. However, multiple nodes sending data at the same time to a common aggregator node is a possible scenario, so we tested the performance for cases where two nodes sent data simultaneously to a single aggregator node on the waist. The results are shown in Table 6 for 802.15.4. The results are averaged over 3 people, however all the people were sitting for this experiment. Also, one of the sending nodes was placed at the chest, while the other was at the ankle. The results can be compared with the average goodput for a single node case for 802.15.4. Averaged over all node positions, activities and people, the 802.15.4 goodput (single node case) was $12.73 \mathrm{kbps}$ in the case of 10 byte packets and $79.48 \mathrm{kbps}$ in the case of 100 byte packets. This shows that going from 1 to 2 nodes sending simultaneously reduced the aggregate goodput by $\sim 55 \%$ for 10 byte packets and $23 \%$ for 100 byte packets.

Similarly, we can look at the corresponding results for BT in Table 7. Again, this can be compared against the case of a single node sending data for which the goodput was $10.45 \mathrm{kbps}$ (10 byte packets) and $94.17 \mathrm{kbps}$ (100 byte packets). Hence going from 1 to 2 sending nodes results in a drop in goodput of $\sim 8 \%$ for 10 byte packets and $\sim 12 \%$ for 100 byte packets.
Table 6 Goodput and packet success rates for 802.15.4 when 2 nodes simultaneously send to a node on the waist

\begin{tabular}{|c|c|c|c|c|}
\hline \multirow[b]{2}{*}{$\begin{array}{l}\text { Node } \\
\text { position }\end{array}$} & \multicolumn{2}{|c|}{10 byte packets } & \multicolumn{2}{|c|}{100 byte packets } \\
\hline & $\begin{array}{l}\text { Goodput } \\
\text { (kbps) }\end{array}$ & $\begin{array}{l}\text { Success } \\
\text { rate }(\%)\end{array}$ & $\begin{array}{l}\text { Goodput } \\
\text { (kbps) }\end{array}$ & $\begin{array}{l}\text { Success } \\
\text { rate }(\%)\end{array}$ \\
\hline Ankle & 2.5 & 40.67 & 16.43 & 49.63 \\
\hline Chest & 3.2 & 51.33 & 45.27 & 74.90 \\
\hline Overall & 5.7 & 46.65 & 61.70 & 68.17 \\
\hline \multicolumn{5}{|c|}{$\begin{array}{c}\text { Table } 7 \text { Goodput and packet success rates for BT when } 2 \\
\text { nodes simultaneously send to a node on the waist }\end{array}$} \\
\hline & \multicolumn{2}{|c|}{10 byte packets } & \multicolumn{2}{|c|}{100 byte packets } \\
\hline $\begin{array}{l}\text { Node } \\
\text { position }\end{array}$ & $\begin{array}{l}\text { Goodput } \\
\text { (kbps) }\end{array}$ & $\begin{array}{l}\text { Success } \\
\text { rate }(\%)\end{array}$ & $\begin{array}{l}\text { Goodput } \\
\text { (kbps) }\end{array}$ & $\begin{array}{l}\text { Success } \\
\text { rate }(\%)\end{array}$ \\
\hline Ankle & 4.8 & 100 & 37.2 & 100 \\
\hline Chest & 4.9 & 100 & 45.5 & 100 \\
\hline Overall & 9.7 & 100 & 82.7 & 100 \\
\hline
\end{tabular}

\subsection{IEEE 802.15.4 results with automatic retransmission}

All the results presented above were without any retransmissions in 802.15.4. Adding automatic retransmissions would increase the success rate, but would lower the goodput due to the signaling overhead. The results for this case are shown in Table 8 for a single sender node for the cases of ankle to waist and waist to ankle. This experiment was carried out on only one person who was sitting for this experiment. The results for the case without retransmission are also shown for ease of comparison. The results clearly show that adding retransmissions to 802.15.4 increases the packet success rate to $100 \%$, albeit at the expense of lowered goodput and higher average latency.

Table 8 Goodput, success rate and average latencies with and without retransmission in 802.15.4

\begin{tabular}{l|llll}
\hline & \multicolumn{3}{|c}{ Ankle to waist } & \multicolumn{2}{c}{ Waist to ankle } \\
& $\begin{array}{l}\text { With } \\
\text { retx }\end{array}$ & $\begin{array}{c}\text { Without } \\
\text { retx }\end{array}$ & $\begin{array}{c}\text { With } \\
\text { retx }\end{array}$ & $\begin{array}{c}\text { Without } \\
\text { retx }\end{array}$ \\
\hline lO byte packets & & & & \\
Goodput (kbps) & 10.33 & 11.88 & 9.7 & 13.37 \\
Success rate (\%) & 100 & 90.12 & 100 & 90.03 \\
Latency (ms) & 8.13 & 7.17 & 8.41 & 5.24 \\
\hline lo0 byte packets & & & & \\
Goodput (kbps) & 45.89 & 78.36 & 57.43 & 80.55 \\
Success rate (\%) & 100 & 85.62 & 100 & 87.94 \\
Latency (ms) & 18.08 & 8.47 & 14.06 & 8.49 \\
\hline
\end{tabular}




\subsection{Summary data}

To provide a final snapshot of all the data, we look at the best values of the goodput and packet success rates across different activities, node positions and people and present a summary in Table 9 for 802.15.4. For e.g. the best activity was standing which was determined based on averaging the values across all people and node positions and then comparing the performance for the different activities of sitting, standing and walking. We also look at the average values across all the data we collected. Note that the data presented in Table 9 includes only the data for 802.15.4 with no retransmissions. The data show that the average goodput for on-body communication across different sets of activities, node positions and people is $12.82 \mathrm{kbps}$ and $80.02 \mathrm{kbps}$ for 10 byte and 100 byte packets respectively, which represents $78 \%$ and $84 \%$ of the baseline case.

Table 9 Summary data for 802.15.4

\begin{tabular}{|c|c|c|c|c|}
\hline & \multicolumn{2}{|c|}{10 byte packets } & \multicolumn{2}{|c|}{100 byte packets } \\
\hline & $\begin{array}{c}\text { Goodput } \\
\text { (kbps) }\end{array}$ & $\begin{array}{l}\text { Success } \\
\text { rate }(\%)\end{array}$ & $\begin{array}{c}\text { Goodput } \\
\text { (kbps) }\end{array}$ & $\begin{array}{l}\text { Success } \\
\text { rate }(\%)\end{array}$ \\
\hline Baseline & 16.43 & 98.94 & 94.63 & 99.78 \\
\hline $\begin{array}{l}\text { Best activity } \\
\text { (standing) }\end{array}$ & 13.92 & 94.82 & 86.48 & 91.62 \\
\hline $\begin{array}{l}\text { Best node } \\
\text { position (chest) }\end{array}$ & 15.30 & 97.69 & 92.95 & 98.68 \\
\hline $\begin{array}{l}\text { Best person } \\
\text { (Person } 1)\end{array}$ & 13.24 & 93.1 & 84.58 & 92.40 \\
\hline $\begin{array}{l}\text { Average across } \\
\text { all data }\end{array}$ & 12.82 & 91.5 & 80.02 & 86.95 \\
\hline
\end{tabular}

The summary data for Bluetooth is shown in Table 10. Since the packet success rate for Bluetooth was $\sim 100 \%$ due to automatic retransmissions, that data is not provided in the table. We also look at the average across all the data we collected. This shows that the average goodput for on-body Bluetooth communication is $84 \%$ and $86 \%$ of the baseline case for 10 byte and 100 byte packets respectively.

Table 10 Summary data for BT

\begin{tabular}{l|ll}
\hline & $\begin{array}{c}\text { 10 byte packets } \\
\text { (Goodput in kbps) }\end{array}$ & $\begin{array}{c}\text { 100 byte packets } \\
\text { (Goodput in kbps) }\end{array}$ \\
\hline Baseline & 12.36 & 115.03 \\
\hline $\begin{array}{l}\text { Best activity } \\
\text { (standing) }\end{array}$ & 11.01 & 104.71 \\
$\begin{array}{l}\text { Best node position } \\
\text { (ear) }\end{array}$ & 10.50 & 101.32 \\
$\begin{array}{l}\text { Best person (Person } \\
\text { 1) }\end{array}$ & 10.74 & 105.24 \\
$\begin{array}{l}\text { Average across all } \\
\text { cases }\end{array}$ & 10.44 & 99.09 \\
\hline
\end{tabular}

\subsection{Radio co-existence}

We examine the performance of each of the radios when the two types of radios are operated simultaneously. Two experiments were conducted to evaluate their coexistence. In the first one, a $\mathrm{BT}$ radio was placed on the ear sending data to a BT receiver on the waist. At the same time an 802.15.4 node was placed on the ankle sending data to an 802.15.4 receiver on the waist. The second experiment interchanged the positions of the BT and 802.15.4 radios on the ear and ankle. This experiment was carried out on only one person and the person was sitting. The results from the two experiments are shown in Table 11. This clearly shows that 802.15.4 takes a much bigger hit in performance than Bluetooth.

Table 11 Goodput (in kbps) for BT and 802.15.4 radios when they simultaneously send data

\begin{tabular}{l|llll}
\hline & \multicolumn{3}{|c}{ Experiment 1 } & \multicolumn{3}{c}{ Experiment 2 } \\
$\begin{array}{l}\text { Packet } \\
\text { size }\end{array}$ & $\begin{array}{l}\text { BT (ear to } \\
\text { waist) }\end{array}$ & $\begin{array}{l}15.4 \text { (ankle } \\
\text { to waist) }\end{array}$ & $\begin{array}{l}\text { BT (ankle } \\
\text { to waist) }\end{array}$ & $\begin{array}{l}15.4 \text { (ear } \\
\text { to waist) }\end{array}$ \\
\hline $\begin{array}{l}10 \text { bytes } \\
100 \text { bytes }\end{array}$ & 12.31 & 1.9 & 9.38 & 0.4 \\
\hline
\end{tabular}

\section{DISCUSSION OF RESULTS}

Looking at the results from the 802.15.4 and Bluetooth experiments, we would like to highlight the following points. First, it seems that in general Bluetooth has consistently higher success rates than 802.15.4 especially in the on-body experiments. This is due to the fact that the Bluetooth MAC automatically incorporates acknowledgement and retransmissions, supports the automatic selection of different levels of forward error correction based on BER measurements (DM versus DH packet types), and leverages channel diversity through frequency hopping. These factors also give Bluetooth an advantage over 802.15.4 in combating on-body communication effects which can be seen from the consistent goodput and success rates across different node locations and activities. When examining 802.15.4 success rates for on-body configurations, we notice a major reduction from the off-body baseline experiment. To improve the success rate, we enabled acknowledgements and retransmission on 802.15.4 which resulted in achieving a 100\% success rates. However, this came at the expense of much lower goodput as can been seen in Table 8. Moreover, 802.15.4 seemed to have more erratic performance than Bluetooth. For e.g. the left waist to waist performance while sitting was much worse for 100 byte packets (see Figure 1) or the latency for ear to waist communication for the 10 byte packets was much smaller than in Table 3 . Such variability in performance would make it harder to design reliable applications.

Second, when we examine the different packet sizes, it is clear that both radios show better goodput when using larger packets. This is to be expected due to the reduction in packet overhead as packet sizes grow. However, when examining the actual goodput measured and comparing it to the maximum, we find a large discrepancy in both radios. It is worth mentioning that Bluetooth packets can occupy different numbers of $.625 \mathrm{msec}$ slots $(1,3$, and 5). Table 12 shows the maximum allowed packet size (after subtracting required headers) for each configuration, and the 
maximum throughput achievable given our 10, 100 and 300 packet sizes. We suspected that the Bluetooth MAC was not optimizing the packet type (which is automatically selected), and ran another set of experiments where we forced it to use a specific packet type by restricting the allowed types during connection establishment. This resulted in increasing the goodput to 12.3 kbps and $117 \mathrm{kbps}$ for the 10 and 100 byte experiments respectively, which are considerable improvements, yet lower than the maximum expected. We believe some of this discrepancy can be attributed to the inefficiency of the OS in supporting back to back transmissions.

Table 12 Maximum throughput given different packet types for Bluetooth

\begin{tabular}{l|lll}
\hline $\begin{array}{l}\text { Number } \\
\text { of slots }\end{array}$ & $\begin{array}{l}\text { Max } \\
\text { Payload }(B)\end{array}$ & $\begin{array}{l}\text { Selected } \\
\text { packet } \\
\text { size(B) }\end{array}$ & $\begin{array}{l}\text { Max throughput (kbps) } \\
\text { (assuming selected packet } \\
\text { size \& symmetric link) }\end{array}$ \\
\hline 1 & 11 & 10 & 64 \\
3 & 167 & 100 & 213 \\
5 & 323 & 300 & 384 \\
\hline
\end{tabular}

Finally, energy consumption is an important factor to consider when comparing these two radios in an on-body environment, given the tight constraints on battery size in wearable devices. We did not perform power measurements on these platforms since the Imote1 platform is outdated and there are newer chipsets in the market that have lower power consumption. We plan to investigate the power consumption in our future work. For the sake of discussion, we compiled a table included in the Appendix that shows the high level power consumption data from some representative data sheets (CC2420 for 802.15.4 [17] and CSR BlueCore4 [19] for Bluetooth). In the 802.15.4 case, the Tx and $\mathrm{Rx}$ current consumption is reported in the sheet, whereas in Bluetooth it is a bit more complicated. The datasheet reports average current consumption for different configurations. Since maintaining the connection will result in sending LM packets over the link, even if data is not being exchanged the radio will still consume power. For Bluetooth we show 4 configurations. The first shows the average current consumption for ACL with no data transfer (ACL link is connected, no data). The second configuration assumes file transfer (115 kbps) over the ACL link. The third shows the peak Tx and Rx current consumption and the fourth assumes the link is in sniff mode (low power), where a packet is exchanged every 1.28 and $0.04 \mathrm{sec}$ respectively (node sleeps periodically for $1.28 / 0.04 \mathrm{sec}$ ). The most relevant comparison while sending data is the ACL with file transfer in BT versus the Tx \& Rx for 802.15.4. In this case, if we consider the send and receive current consumption, the two radios are very comparable. However, looking at the overhead of a connection compared to 802.15.4, even with no data transfer in Bluetooth, we have a much higher current consumption than the idle current in 802.15.4 (10x - 30x higher). To improve the power efficiency in Bluetooth, one can certainly leverage the sniff mode, which would be as low as the idle mode in 802.15.4. However, sniff mode in Bluetooth is much more restricted than the idle mode in 802.15.4 since the node will be sleeping for 1.28 seconds to get to this low current consumption, which will increase the latency of communication compared to the 802.15 .4 case. One can certainly reduce the sniff interval in Bluetooth, however, the current consumption will increase as a result (reaching $2.4 \mathrm{~mA}$ at $40 \mathrm{msec}$ intervals).

\section{CONCLUSIONS AND FUTURE WORK}

Based on the experiments we conducted, it seems that Bluetooth has overall superior performance for on-body networks, especially in the consistency of the results and is more resilient to changes in activities, node positions and on-body effects in general. For larger packets, Bluetooth provides better goodput and packet success rates. For smaller packets, IEEE 802.15.4 was actually superior in terms of the goodput. However, when comparing the goodput at similar packet success rates (by turning on retransmissions), Bluetooth delivers better goodput across both packet types. On the other hand, IEEE 802.15.4 has about onehalf to one-third power consumption of Bluetooth, which means it may be more suitable for low data rate applications, especially where latency requirements are not as stringent (IEEE 802.15.4 suffers from relatively longer channel fades, so high latencies could be possible for some packets). However, it should be noted that Bluetooth supports "Sniff" mode which would reduce its power consumption while supporting data transfer at lower rates (e.g. CSR BlueCore4 [19] reports $40 \mathrm{msec}$ sniff @ $2.11 \mathrm{~mA}$ and $1.28 \mathrm{sec}$ sniff @ $0.42 \mathrm{~mA}$ ), so actual measurements would be necessary to evaluate the energy impact. We leave that as future work.

Bluetooth also had much better performance when multiple nodes sent data simultaneously to the destination. IEEE 802.15.4 had a drastic reduction in performance compared to the single node case, which may also be due to the fact that we used BMAC rather than the real IEEE 802.15.4 MAC. It would be interesting to run similar tests with the real IEEE 802.15.4 MAC to see if that is indeed the case. Additionally, more results are needed for the performance of IEEE 802.15.4 with automatic retransmissions to have a direct and fair comparison with Bluetooth.

Finally, simultaneous operation of the two types of radios clearly showed that IEEE 802.15.4 performance degrades far more than Bluetooth performance. With the proliferation of various types of wireless technologies, this is a serious concern that would need to be addressed for IEEE 802.15.14. In general, we would need to consider interference detection and mitigation strategies for the radios if body area networks are to become a reality given that such networks would constantly come in and out of range of each other.

\section{ACKNOWLEDGMENTS}

Our thanks to the multiple volunteers who let us test the performance of the radios when placed on them and the anonymous reviewers for their helpful comments.

\section{REFERENCES}

[1] T. Y. Chui, F. Thaler, and W. G. Scanlon, "A Novel Channel Modeling Technique for Performance Analysis of Bluetooth Baseband Packets". In IEEE International Conference on Communications, Volume 1, Issue 1, 2002 Page(s):308 312.

[2] Roelens, L. Van den Bulcke, S. Joseph, W. Vermeeren, G. Martens, L., "Path loss model for wireless narrowband communication above flat phantom". In IEEE Electronics Leters, 42 (1), pg 10-11, Jan. 2006. 
[3] N. Kuster and Q. Balzano, "Energy Absorption Mechanism by Biological Bodies in the Near Field of Dipole Antennas above $300 \mathrm{MHz}$ ". In IEEE Transactions on vehicular technology, Vol. 41, No. 1, Feb 1992.

[4] A. J. Johansson, "Wave-Propagation from medical implantsinfluence of body shape on radiation pattern", In Proceedings of the $2^{\text {nd }}$ Joint EMBS/BMES Conference, Houston, TX, USA, Oct 2002.

[5] S. K. S. Gupta, S. Lalwani, Y. Prakash, E. Elsharawy, L. Schwiebert, "Towards a propagation model for wireless biomedical applications". In IEEE Intl. Conference on Communications, Vol 3, pg 1992 - 1997, May 2003.

[6] N. Golmie, D. Cypher, and O. Rebala, "Performance evaluation of low rate WPANs for Medical Applications".

[7] J. Werb, M. Newman, V. Berry, and S. Lamb, "Improved Quality of Service in IEEE 802.15.4 Mesh Networks". In International Workshop on Wireless and Industrial Automation. March, 2005.

[8] A. Sikora, "Compatibility of IEEE 802.15.4 (ZigBee) with IEEE802.11 (WLAN), Bluetooth, and Microware Ovens in 2.4 GHz ISM-Band". Technical report.

[9] G.-Z. Yang. "Ubimon - ubiquitous monitoring environment for wearable and implantable sensors". Available from: http://www.doc.ic.ac.uk/vip/ubimon/research/index.html.

[10] G. Virone, A. Wood, L. Selavo, Q. Cao, L. Fang, T. Dozn, Z. He, and J. Stankovic, "An advanced wireless sensor network for health monitoring”. In Trans-disciplinary Conference on Distributed Diagnosis and Home Healthcare (D2H2), April 2006.

[11] D. Husemann, IBM Personal care connect. Available from: http://www.zurich.ibm.com/pcc/index.html.

[12] V. Shnayder, B. Chen, K. Lorincz, R. R. F. Fulford-Jones, and M. Welsh, "Sensor Networks for Medical Care". Technical Report TR-08-05, Division of Engineering and Applied Sciences, Harvard University, 2005.

[13] A. Natarajan, M. Motani, B. de Silva, K. Yap, and K. C. Chua, "Investigating Network Architectures for Body Sensor Networks", In Proc of HealthNet'07, June, 2007, San Juan, Puerto Rico, USA.

[14] L. Nachman, R. Kling, R. Adler, J. Huang, and V/ Hummel, "The Intel Mote platform: A Bluetooth-based sensor network for industrial monitoring", International Symposium on information processing in sensor networks, 2005.
[15] R. Adler, et al., "Demo Abstract: Intel Mote 2: An Advanced Platform for Demanding Sensor Network Applications," Proceedings of the 3rd International Conference on Embedded Networked Sensor Systems, SenSys'05, San Diego, CA, Nov. 2005.

[16] TinyOS Community Forum II An open-source OS for the networked sensor regime. http://www.tinyos.net/.

[17] Chipcon, Texas Instruments. CC2420 data sheet. http://focus.ti.com/docs/prod/folders/print/cc2420.html.

[18] J. Polastre, J. Hill, and D. Culler, "Versatile Low Power Media Access for Wireless Sensor Networks", SenSys, November 3-5, 2004.

[19] CSR. BlueCore4 data sheet. http://www.csrsupport.com/download/1539/BlueCore4External\%20Datasheet\%20BC417143B-ds-001Pg.pdf.

\section{APPENDIX}

Current consumption for various IEEE 802.15.4 and Bluetooth configurations

\begin{tabular}{|l|l|l|}
\hline Type & $\begin{array}{l}802.15 .4 \\
m A\end{array}$ & $\begin{array}{l}\text { Bluetooth } \\
m A\end{array}$ \\
\hline Tx & 17.4 & \\
\hline Rx & 19.7 & \\
\hline Idle & 0.426 & \\
\hline ACL with no data transfer (Master) & & 4.6 \\
\hline ACL with no data transfer (Slave) & & 17 \\
\hline ACL with file transfer (Master) & & 10.3 \\
\hline ACL with file transfer (Slave) & & 24.7 \\
\hline Peak Tx & & 51.5 \\
\hline Peak Rx & & 39 \\
\hline Sniff @ 1.28 sec (Master) & & 0.37 \\
\hline Sniff @ 40 msec (Master & & 2.4 \\
\hline
\end{tabular}

